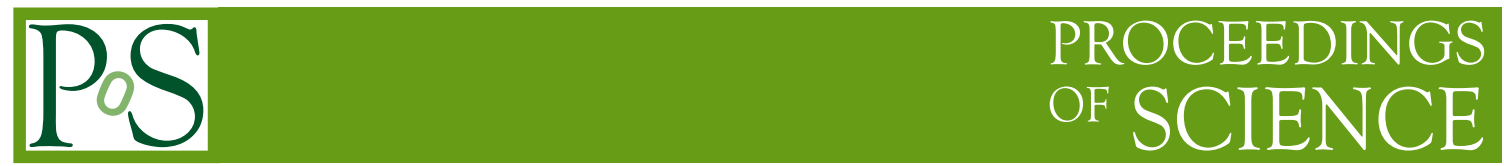

\title{
Introduction to the High Energy cosmic-Radiation Detection (HERD) Facility onboard China's Future Space Station
}

Shuang-Nan Zhang ${ }^{\star a, c}{ }^{\circ}$ Oscar Adriani ${ }^{l}$, Sebastiano Albergo ${ }^{f}$, Giovanni Ambrosi ${ }^{g}$, Qi $\mathrm{An}^{B}$, Philippe Azzarello ${ }^{h}$, Yonglin $\mathrm{Bai}^{b}$, Tianwei Bao ${ }^{a}$, Paolo Bernardini ${ }^{q}$, Bruna Bertucci $^{m}$, Xiaojun $\mathrm{Bi}^{a}{ }^{a}$, Massimo Bongi ${ }^{l}$, Sergio Bottai ${ }^{o}$, Weiwei $\mathrm{Cao}^{b}{ }^{b}$,Zhen Cao $^{a}$,Junying Chai ${ }^{a c}$, Jin Chang ${ }^{C}$, Guoming Chen $^{a}$, Y Chen $^{u}$, Zhen Chen ${ }^{b}, \mathbf{X} . \mathbf{H}$. Cui $^{\nu}$,Z.G. Dai ${ }^{u}$, Raffaello D'Alessandro ${ }^{l}$, Margherita Di Santo ${ }^{k}$, Yongwei Dong ${ }^{a}$,

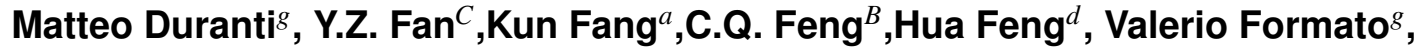
Piergiorgio Fusco $^{r}$, Jiarui Gao ${ }^{b}$, Fabio Gargano ${ }^{n}$, Nicola Giglietto ${ }^{r}$, Q.B. Gou ${ }^{a}$, Y.Q. Guo $^{a}$, H.H. He ${ }^{a}$, H.B. Hu ${ }^{a}$,Peng Hu${ }^{a c}$, G.S. Huang ${ }^{b}$,J. Huang ${ }^{a}$, Y.F. Huang ${ }^{u}, \mathbf{H} \mathbf{L i}^{a}$, Ran $\mathbf{L i}^{b}$,Yong Li ${ }^{b}$, Z. Li ${ }^{w}$, E.W. Liang ${ }^{e}$, Sujie Lin ${ }^{a}$, H. Liu ${ }^{a}$, Hongbang Liu ${ }^{e}$, J.B. Liu ${ }^{B}$, S.B. Liu $^{B}$, S.M. Liu ${ }^{C}, \mathbf{X i n}$ Liu $^{a c}$, Francesco Loparco ${ }^{r}$, Junguang Lyu ${ }^{a}$, Giovanni Marsella ${ }^{q}$, Mario Nicola Mazziottai ${ }^{n}$, Ivan De Mitri ${ }^{q}$, Nicola Mori ${ }^{o}$, Paolo Papini ${ }^{o}$, Mark Pearce ${ }^{s}$, Wenxi Peng ${ }^{a}$, Martin Pohl ${ }^{h}$, Zheng Quan ${ }^{a}$, Felix Ryde ${ }^{s}$, Dalian Shi ${ }^{b}$, Meng Su ${ }^{t}$, X.L. Sun $^{a}$, Xin Sun $^{b}$, Antonio Surdo $^{k}$, Z.C. Tang ${ }^{a}$, Elena Vannuccini ${ }^{o}$, Roland Walter ${ }^{h}$, Bingbing Wang ${ }^{a}$, Bo Wang ${ }^{b}$, J.C. Wang ${ }^{x}$, J.M. Wang ${ }^{a}$, Junjing Wang $^{a c}$,Le Wang ${ }^{b}$, Ruijie Wang ${ }^{a}$, X.L Wang ${ }^{B}$,X.Y. Wang ${ }^{u}$,Zhigang Wang ${ }^{a}$, D.M. Wei ${ }^{C}$,Bobing Wu $^{a}$, J.

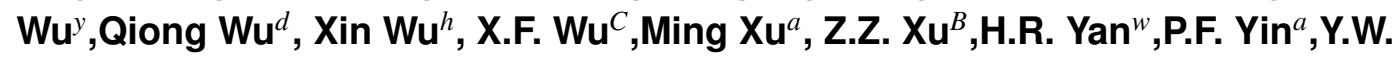

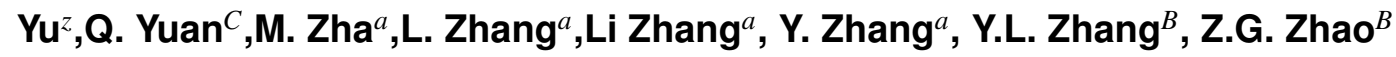


${ }^{a}$ Key Laboratory of Particle and Astrophysics, Institute of High Energy Physics, Chinese Academy of Sciences, Beijing, China

${ }^{b} X i$ 'an Institute of Optics and Precision Mechanics of CAS, Xi'an, Shaanxi, China

${ }^{c}$ University of Chinese Academy of Sciences, Beijing, China

${ }^{d}$ Department of Engineering Physics and Center for Astrophysics, Tsinghua University, Beijing, China

${ }^{e}$ Department of Physics, Guangxi University, Nanning, China

${ }^{f}$ Istituto Nazionale di Fisica Nucleare, Sezione di Catania, Catania, Italy

${ }^{g}$ Istituto Nazionale di Fisica Nucleare, Sezione di Perugia, Perugia, Italy

${ }^{h}$ Nuclear and Corpuscular Physics Department, University of Geneva, Geneva, Switzerland

${ }^{i}$ University of Pisa, Pisa, Italy

${ }^{j}$ Istituto Nazionale di Fisica Nucleare, Sezione di Trento, Trento, Italy

${ }^{k}$ Istituto Nazionale di Fisica Nucleare, Sezione di Lecce, Lecce, Italy

${ }^{l}$ University of Florence and Istituto Nazionale di Fisica Nucleare, Sezione di Firenze,, Firenze, Italy

${ }^{m}$ University of Perugia and Istituto Nazionale di Fisica Nucleare, Sezione di Perugia, Perugia, Italy

${ }^{n}$ Istituto Nazionale di Fisica Nucleare, Sezione di Bari, Bari, Italy

${ }^{o}$ Istituto Nazionale di Fisica Nucleare, Sezione di Firenze, Firenze, Italy

${ }^{p}$ ISDC, University of Geneva, Geneva, Switzerland

${ }^{q}$ Universita' del Salento and Istituto Nazionale di Fisica Nucleare, Sezione di Lecce, Lecce, Italy

${ }^{r}$ Dipartimento di Fisica dell'Univerisit'a del Politecnico di Bari, and Istituto Nazionale di Fisica Nucleare, Bari, Italy

${ }^{s}$ KTH Royal Institute of Technology, Stockholm, Sweden

${ }^{t}$ Hong Kong University

${ }^{u}$ School of Astronomy and Space Science, Nanjing University, Nanjing, China

${ }^{v}$ National Astronomical Observatories, Chinese Academy of Sciences, Beijing, China

${ }^{w}$ Department of Astronomy, Peking University, Beijing, China

${ }^{x}$ Yunnan Astronomical Observatory, Chinese Academy of Sciences, Kunming, China

${ }^{y}$ Department of Physics, China University of Geosciences, Wuhan, China

${ }^{z}$ Institute of Astrophysics, Central China Normal University, Wuhan, China

${ }^{A}$ Department of Astronomy, Yunan University, Kunming, China

${ }^{B}$ Department of Modern Physics, University of Science and Technology of China, Hefei, China

${ }^{C}$ Purple Mountain Observatory, Chinese Academy of Sciences, Nanjing, China 
The High Energy cosmic-Radiation Detection (HERD) facility is one of several space astronomy payloads onboard China's Space Station, which is planned for operation starting around 2025 for about 10 years. The main scientific objectives of HERD are searching for signals of dark matter annihilation products, precise cosmic electron (plus positron) spectrum and anisotropy measurements up to $10 \mathrm{TeV}$, precise cosmic ray spectrum and composition measurements up to the knee energy, and high energy gamma-ray monitoring and survey. HERD is composed of a 3-D cubic calorimeter (CALO) surrounded by microstrip silicon trackers (STKs) from five sides except the bottom. CALO is made of about 7,500 cubes of LYSO crystals, corresponding to about 55 radiation lengths and 3 nuclear interaction lengths, respectively. The top STK microstrips of six $\mathrm{X}$-Y layers are sandwiched with tungsten converters to make precise directional measurements of incoming electrons and gamma-rays. In the baseline design, each of the four side STKs is made of only three layers microstrips. All STKs will also be used for measuring the charge and incoming directions of cosmic rays, as well as identifying back scattered tracks. With this design, HERD can achieve the following performance: energy resolution of $1 \%$ for electrons and gamma-rays beyond $100 \mathrm{GeV}$ and $20 \%$ for protons from $100 \mathrm{GeV}$ to $1 \mathrm{PeV}$; electron/proton separation power better than $10^{-5}$; effective geometrical factors of $>3 \mathrm{~m}^{2} \mathrm{sr}$ for electron and diffuse gamma-rays, $>2 \mathrm{~m}^{2} \mathrm{sr}$ for cosmic ray nuclei. R\&D is under way for reading out the LYSO signals with optical fiber coupled to image intensified IsCMOS and CALO prototype of 250 LYSO crystals.

35th International Cosmic Ray Conference - ICRC2017

10-20 July, 2017

Bexco, Busan, Korea

\footnotetext{
* Speaker.

$\dagger$ This work is supported by the National Natural Science Foundation of China, Grant No.11327303 and Grant No.11473028; Key Research Program of Frontier Sciences, CAS, Grant NO. QYZDY-SSW-SLH008; the International Partnership Program of Chinese Academy of Sciences, Grant No. 113111KYSB20160053.
} 


\section{INTRODUCTION}

It is well established that neutral, cold/warm and non-baryonic dark matter (DM) dominates the total matter content in the universe. Weakly Interacting Massive Particles[1] (WIMPs) are well motivated candidates of DM particles. One way to detect WIMPs is to search for its annihilation/decay products, which may lead to characteristic features in the observed spectra of cosmic electrons (plus positron) or gamma-ray spectra. Some circumstantial evidence or hints of anomalies have been reported[2, 3, 4]; however, astrophysical sources like pulsars and pulsar wind nebulae can also contribute to these results. Future more precise measurements at higher energies are still needed.

The steepening of the primary cosmic ray (CR) spectrum around PeV, the so-called "knee" structure is a classic problem in CR physics since its discovery in 1958, but still unresolved[5]. Ground-based extensive air shower experiments[6, 7, 8] continue to make progress [9]; however, these experiments have difficulties in making composition-resolved high-energy resolution measurements of the fine structure of the "knee". On the other hand, experiments based on balloons[14, 16], satellites[20], or the international space station[13] can measure the particle energy and charge directly; however, these experiments suffer from small geometrical factor and limited energy range to make statistically meaningful measurements of the "knee".

Several generations of wide field of view (FOV) space gamma-ray telescopes in the GeV energy regime and ground based narrow FOV gamma-ray telescopes in hundreds of GeV energy regimes have discovered several new populations of extreme astrophysical objects, which allow deeper understanding of the laws of nature under extreme physical conditions only available in cosmic laboratories. In particular the wide FOV space gamma-ray telescopes often provide crucial guidance to the observations of the ground-based narrow FOV telescopes. Unfortunately, the much more powerful ground-based Cherenkov Telescope Array (CTA) currently under development may not have the much needed guidance from a space wide FOV gamma-ray telescope, once the Fermi satellite stops operations. A new wide FOV space gamma-ray telescope is urgently needed to replace Fermi.

In order to address the above major problems in fundamental physics and astrophysics, the High Energy cosmic-Radiation Detection (HERD) facility has been planned as one of several space astronomy payloads onboard China's space station, which is planned for operation starting around 2025 for about 10 years. In this paper, we describe the scientific drivers of the design of HERD, its basic characteristics determined with Monte-Carlo simulations, as well as ongoing R\&D efforts in developing HERD.

\section{HERD Scientific Objectives, Requirements and Baseline Design}

The primary scientific objectives of HERD are: (1) searching for signatures of the annihilation products of dark matter particles in the energy spectra and anisotropy of high energy electrons and gamma-rays from $500 \mathrm{MeV}$ to $10 \mathrm{TeV}$; (2) measuring precisely and directly the energy spectra and composition of primary cosmic rays from $10 \mathrm{GeV}$ up to PeV. The secondary scientific objectives of HERD include wide FOV monitoring of the high energy gamma-ray sky from $500 \mathrm{MeV}$ up to 10 $\mathrm{TeV}$ for gamma-ray bursts, active galactic nuclei and Galactic microquasars. Since models of dark 
matter particle annihilations do not yet have strong predictive power, our strategy in the baseline design of HERD is to ensure that the effective geometrical factor, energy range and resolution of HERD meet the requirements for observations of cosmic rays, while maintaining the best possible capability in observing electrons and gamma-rays within the currently available resources for placing HERD on board China's space station. Extensions to the baseline design may be made to increase its effective geometrical factor for gamma-rays (and electrons) with excellent energy and angular resolution.
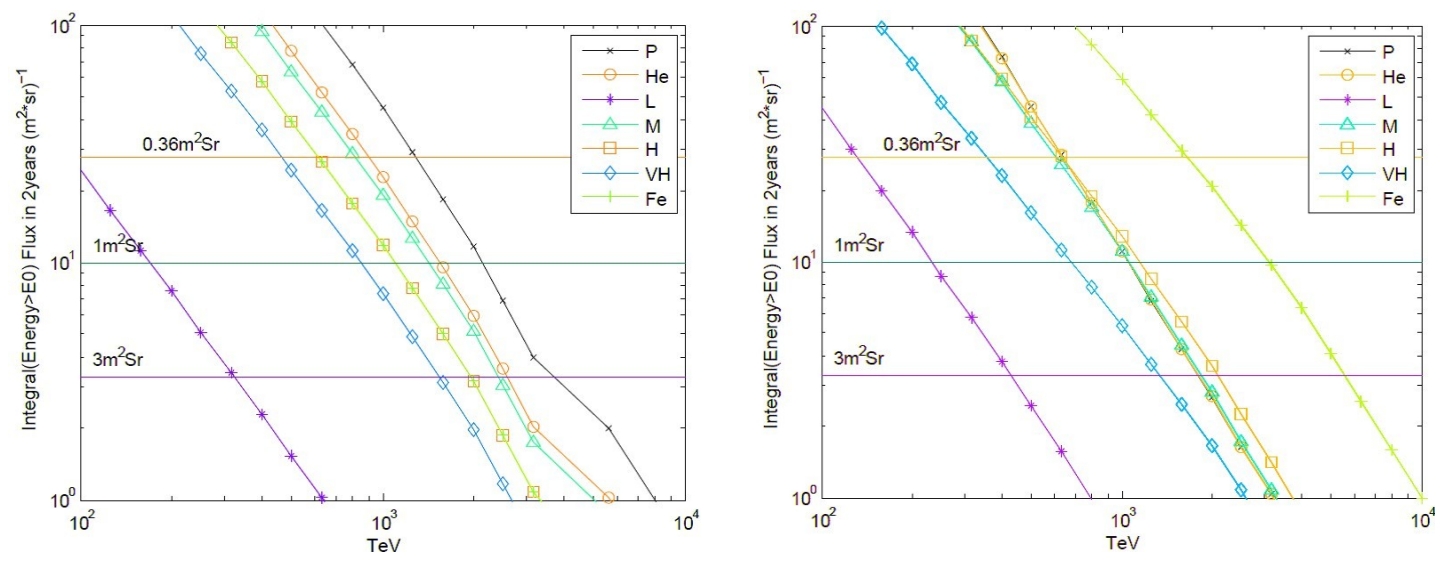

Figure 1: Total number of events per $\mathrm{m}^{2} \mathrm{sr}$ in two years as a function of threshold energy for each of the cosmic ray compositions, predicted with the hard and nonlinear acceleration models[7]. The horizontal lines show the flux required above a certain energy for detecting 10 events in two years with different effective geometrical factor in units of $\mathrm{m}^{2} \mathrm{sr}$. In the left and right panels, cosmic-rays are dominated by protons and iron nuclei, respectively. For convenience we define several groups of elements: " $L$ " with $3 \leq Z \leq 5$, "M" with $6 \leq Z \leq 9$, "H" with $10 \leq Z \leq 19$, "VH" with $Z \geq 20$ but excluding Fe.

In Fig. 1, we show the model predicted fluxes for different compositions of cosmic rays with the hard and nonlinear acceleration models[7]. We considered two extreme cases, i.e., cosmic rays are dominated by protons or iron nuclei, respectively. We conclude that a geometrical factor (with $100 \%$ efficiency) of $\sim 3 \mathrm{~m}^{2} \mathrm{sr}$ is required in order to detect at least 10 events above PeV for all groups of nuclei, except the very rare " $L$ " group with $3 \leq Z \leq 5$, i.e. Lithium, Beryllium and Boron. Our design goal for the calorimeter of HERD is thus simply to achieve an effective geometrical factor of $\sim 3 \mathrm{~m}^{2}$ sr after taking into account the detection and event reconstruction efficiency. To do this, we find that the HERD baseline design with a cubic calorimeter (CALO) of $63 \mathrm{~cm} \times 63 \mathrm{~cm} \times 63 \mathrm{~cm}$ is required, which is made of nearly 7,500 pieces of granulated LYSO crystals of $3 \mathrm{~cm} \times 3 \mathrm{~cm} \times 3 \mathrm{~cm}$ each. From any incident directions, CALO has a minimum stopping power of $55 X_{0}$ and $3 \lambda$, where $X_{0}$ and $\lambda$ are radiation and nuclear interaction lengths, respectively. Such a deep and high granularity calorimeter is also essential for excellent electron-proton separation and energy resolutions of all particles. It also has some directional measurement capability with the reconstructed 3-D showers.

In order to measure the charges and incident directions of cosmic rays, silicon trackers (STKs) are required with a minimum of three layers of silicon micro-strip detectors (SSDs), which can also be used to reject backslash tracks from the showers in CALO. To measure accurately the 


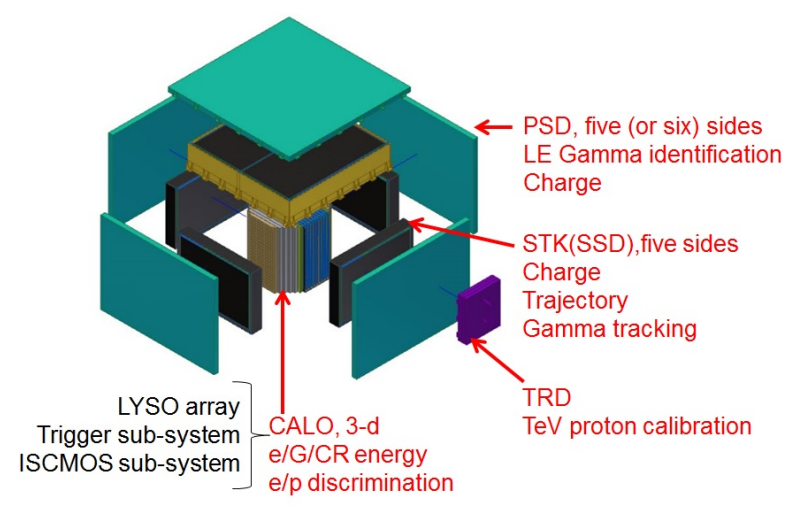

Figure 2: Schematic diagram of the baseline design of HERD. HERD is composed of a 3-D cubic calorimeter (CALO) surrounded by microstrip silicon trackers (STKs) from five sides except the bottom. Then the CALO and STK are covered by the plastic scintillator detector (PSD) from outside. A Transition Radiation Detector (TRD) is located on the lateral side

Table 1: HERD baseline characteristics of CALO and STK

\begin{tabular}{cccccc}
\hline \hline & type & size & $X_{0}, \lambda$ & unit & main functions \\
\hline Top STK & Si strips & $140 \times 140 \mathrm{~cm}^{2}$ & $2 X_{0}$ & $6 \mathrm{x}-\mathrm{y}$ & Charge,track, $\gamma$ converter \\
4-side STK & Si strips & $120 \times 70 \mathrm{~cm}^{2}$ & - & $3 \times-y$ & Charge, nucleon track \\
CALO & $\sim 7500$ & $70 \times 70$ & $55 X_{0}, 3 \lambda$ & $3 \times 3$ & Energy $(\mathrm{e} / \gamma$, nucleon), \\
& cubes & $\times 70 \mathrm{~cm}^{3}$ & & $\times 3 \mathrm{~cm}^{3}$ & e/p separation \\
\hline \hline
\end{tabular}

incident directions of gamma-rays, electron-position pairs should be created and tracked; this can be achieved by adding passive or active conversion materials as shower converters and three more layers of SSDs. In the baseline design of HERD, the top STK is equipped with six layers of SSDs sandwiched with gamma converters, as shown in Fig. 2. The lateral STKs are equipped with three layers of SSDs and no gamma converters. If enough resources are allocated, all the five sides will be covered by the six-layer STK with gamma converter, to ensure the maximum FOV for electrons and gamma-rays. Plastic scintillators surrounding HERD from five sides are needed to reject most low energy charged particles, in order to have maximum efficiency for high energy cosmic rays and electrons, as well as gamma-rays of all energies. Bottom side of the CALO could also be covered by plastic scintillation bars, to improve the capability of particle discrimination. A transition radiation detector is installed on one lateral side of HERD CALO. Unlike the TRD detector in AMS-02 experiment mainly used for particle discrimination, TRD in HERD is designed to perform the cross calibration of $\mathrm{TeV}$ proton and other nuclei by CALO. Two technical solutions of the HERD TRD currently under study are straw tube scheme and MWPC scheme. The HERD baseline characteristics and main functions of its CALO and STKs are listed in Table 1.

\section{Expected Performance of the HERD Baseline Design}

Extensive simulations have been carried out with GEANT4[10] and FLUKA[11], in order 

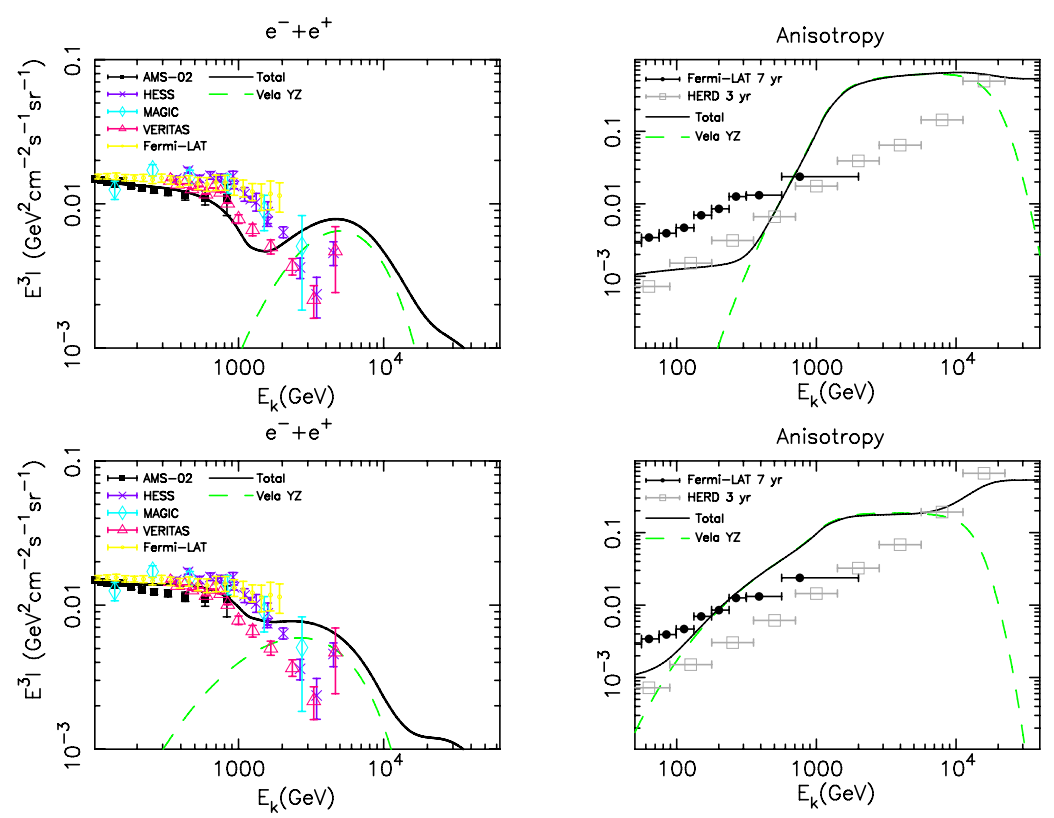

Figure 3: The scenario that Vela $\mathrm{YZ}$ gives dominant contribution to TeV region, with different electron/positron release time. Top graphs: the $e^{+}+e^{-}$spectrum and anisotropy spectrum in the case of an injection age of $1 \mathrm{kyr}$ for Vela YZ. bottom graphs: the same with the top ones but with an injection age of $5 \mathrm{kyr}$ for Vela YZ. The Fermi exclusion data on the anisotropy of the electron flux are shown, together with the expected sensitivity of HERD. The figure is taken from [24].

to evaluate the scientific performance of the HERD baseline design and to optimize the relative weights of each component of HERD within the boundary conditions for accommodating HERD on board China's space station. Since the performance of CALO is key to meeting the scientific goals of HERD, here we only present our simulation results of CALO, by focusing on its effective geometrical factor, energy resolution and e/p separation capability, in order to predict the observed cosmic ray spectra. A key assumption is that an average of 10 photoelectrons can be collected per minimum ionization particle response, which is the design goal of our readout system and already demonstrated in our laboratory test system. For its sensitivity of gamma-ray continuum all sky survey and line observations, certain assumptions are made for its STKs, based on primarily the performance of SSDs of Fermi and AMS02. To simplify the simulations, no mechanical and other supporting structures and materials are included in the simulations. In Table 2, we list the expected HERD baseline performance from Monte-Carlo simulations.

A primary scientific goal for HERD is to measure the high energy electron/positron spectrum up to about $10 \mathrm{TeV}$. The large amount of data accumulation also helps to give a sensitive measurement of the anisotropy of the electron flux. Combined spectrum and anisotropy measurements can show strong indication to the possible nearby electron/positron sources. In Fig. 3 we show the expected electron/positron flux from the assumed nearby supernova remnant Vela YZ. It is shown that Vela YZ may contribute a high energy bump at the electron spectrum. It is also shown that a quite large anisotropy of the electron flux is predicted if such a large flux is generated. The data 
Table 2: HERD baseline performance. Note that in the baseline design, i.e., only the top STK has six layers of SSDs sandwiched with gamma converter, which is expected to deliver an angular resolution of $0.1^{\circ}$ with $\sim 1 \mathrm{~m}^{2}$ sr. In the extended HERD design, all five sides have almost identical STKs as the top STK. It should also be noted, the current HERD STKs can deliver only very poor angular resolution down to the 500 $\mathrm{MeV}$ lower energy limit for gamma-rays; further significant improvements in the STK design are required to enhance its low energy gamma-ray capability.

\begin{tabular}{lll}
\hline \hline Item & Value & Detector \\
\hline \hline Energy range $(\mathrm{e} / \gamma)$ & $10 \mathrm{GeV}-10 \mathrm{TeV} ; 0.5-10 \mathrm{GeV}(\gamma)$ & CALO\&STK \\
Energy range (nucleus) & $30 \mathrm{GeV}-3 \mathrm{PeV}$ & CALO \\
Angular resolution $(\mathrm{e} / \gamma)$ & $0.1 \mathrm{deg} @ 10 \mathrm{GeV}$ & TOP STK \\
Charge measurement (nucleus) & $0.1-0.15 \mathrm{c.u}$. & STK \\
Energy resolution (e) & $1 \% @ 200 \mathrm{GeV}$ & CALO \\
Energy resolution (p) & $20 \% @ 100 \mathrm{GeV}-\mathrm{PeV}$ & CALO \\
e/p separation & $\sim 10^{-6}$ & CALO\&PSD \\
Geometric factor $(\mathrm{e})$ & $>3 \mathrm{~m}^{2} \mathrm{Sr} @ 200 \mathrm{GeV}$ & CALO \\
Geometric factor $(\mathrm{p})$ & $>2 \mathrm{~m}^{2} \mathrm{Sr} @ 100 \mathrm{GeV}$ & CALO \\
\hline \hline
\end{tabular}

on the exclusion limit of the anisotropy from Fermi-LAT is shown in Fig. 3, together with the expected sensitivity of HERD.

In comparison with all previous and other approved missions, the key performance of HERD is its extremely large effective geometrical factor for all types of high energy cosmic radiations, thanks to its very deep 3-D CALO and five-side STKs. In Fig. 4, we show the predicted HERD spectra for protons, helium nuclei, carbon nuclei and iron nuclei, in comparison with all previous direct measurements in space. Clearly HERD will surpass all previous results of directly measured cosmic rays from, e.g., AMS02[13], ATIC-2[14], BESS[15], CREAM,[16, 17] HEAO[18], JACEE[19], PAMELA[20], RUNJOB[21], SOKOL[22] and TRACER[23], with much better statistics and up to much higher energies even beyond $\mathrm{PeV}$ and into the "knee" region. For example, at least ten events will be recorded from $900 \mathrm{TeV}$ to $2 \mathrm{PeV}$ for each specie, which means that the expected energy spectra of most nuclei will be directly extended to the knee range with much smaller error bars than previous direct measurements in space.

With an adequate design of STKs, HERD will also have adequate capability for gamma-ray observations, as shown in Fig. 5. In the post-Fermi era, HERD will be the most sensitive gammaray all-sky survey and transient monitor from $\mathrm{GeV}$ to around $\mathrm{TeV}$, an essential capability to provide triggers and alerts to other multi-wavelength telescopes, such as the future ground based CTA. It is widely anticipated that gamma ray emission lines are the smoking guns for identifying dark matter particle annihilations. As shown in the right panel of Fig. 5, HERD's line sensitivity is far superior to all other missions, due to the combination of its excellent energy resolution, very large effective geometrical factor and high background rejection efficiency.

\section{Key technology of HERD}

A novel method of reading out the LYSO signals by Wavelength Shifting Fiber (WLSF) and 

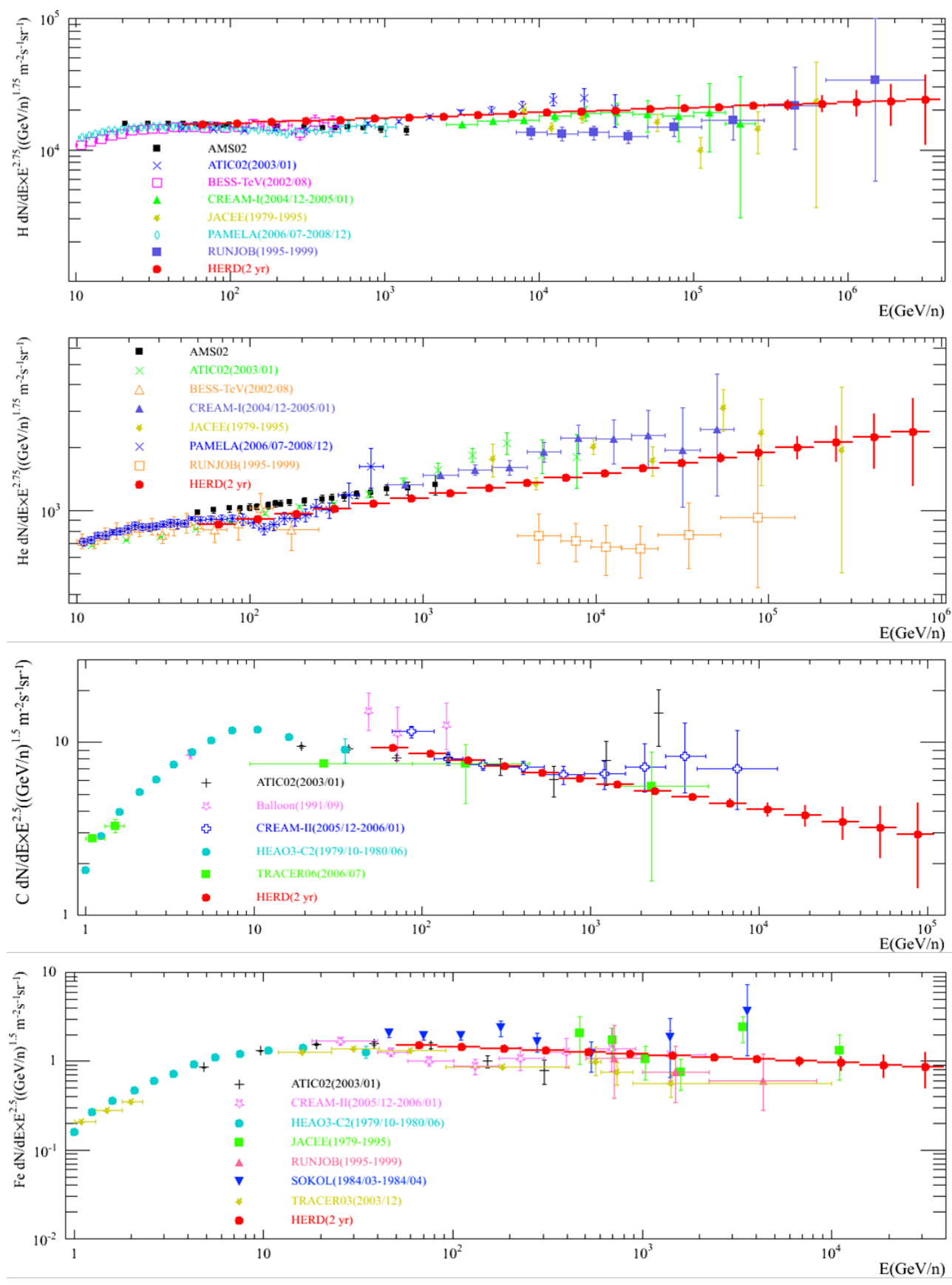

Figure 4: Simulated two-year HERD cosmic ray spectra of protons, heliums, carbons and irons (from top to bottom), in comparison with previous direct measurements in space or at balloon altitudes. The input cosmic ray composition model[12] for the simulation is a combined fitting result from previous measurements. 

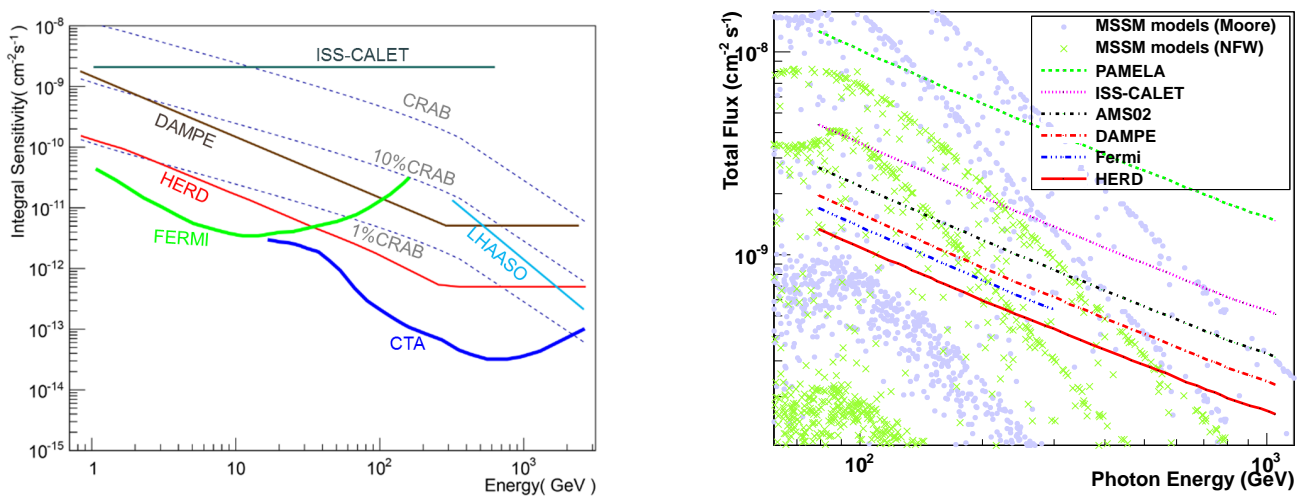

Figure 5: Expected gamma-ray sky survey sensitivity of the extended HERD design, i.e., all five sides are surrounded by the same six-layer STKs with $0.1^{\circ}$ angular resolution across the whole energy band; for the baseline design, the sensitivity is degraded by nearly a factor of 2 . Left: HERD $5 \sigma$ continuum sensitivity for one year observation in comparison with all other missions with gamma-ray observation capability, e.g., ISS-CALET[28], DAMPE and Fermi[25], and including the future CTA[26] and the Large High Altitude Air Shower Observatory (LHAASO)[27]. Right: HERD one-year $5 \sigma$ line sensitivity in comparison with predictions of different dark matter models; the sensitivity lines of other experiments are calculated with the following operation periods: 2006-2016 (PAMELA), 2016-2021 (CALET); 2011-2021 (AMS02), 20162021 (DAMPE), 2008-2018 (Fermi).

image intensified CMOS (IsCMOS) is proposed, which can greatly reduce the complexity of onboard electronics. Linearity of light output of LYSO crystal in the range from $30 \mathrm{MeV}$ to $1 \mathrm{PeV}$ was verified at BEPC E2 by using calibrated electron bundles[29]. So the key technology of HERD is the realization of dynamic range of $10^{7}$ in the IsCMOS system. For the realization of larger dynamic range, each crystal is coupled with three WLSFs. Two fibers are for high and low range IsCMOS systems and the other one is for the trigger system. The three fibers at the crystal end are reshaped into spirals to get the largest contact area with the crystal. Nearly the same number of scintillation photons are absorbed by the three fibers and deposited energy in the crystal is inherited by re-scintillated photons in the fibers.

The high range IsCMOS system and the low range one are distinguished by different gain settings of the image intensifiers. Energy information can be derived from the high range IsCMOS when saturation occurs in the low range IsCMOS. About 20 by 20 CMOS pixels are assigned to one fiber. The sum of charges in these pixels is expected to be linear to the energy deposition in the corresponding crystal. Crosstalk between faculae has to be measured for accurate energy reconstruction.

A beam test on a HERD prototype, composed of an array of $5 * 5 * 10$ LYSO crystals and two ICCD systems, was implemented at CERN SPS in 2015. The main technologies and the reading out scheme of WLSF+ICCD were successfully verified[30]. A beam test on upgraded HERD prototype will be arranged at CERN SPS in October, 2017. Key performances of the CALO, PSD functions and dynamic range of STK electronics will be verified. 

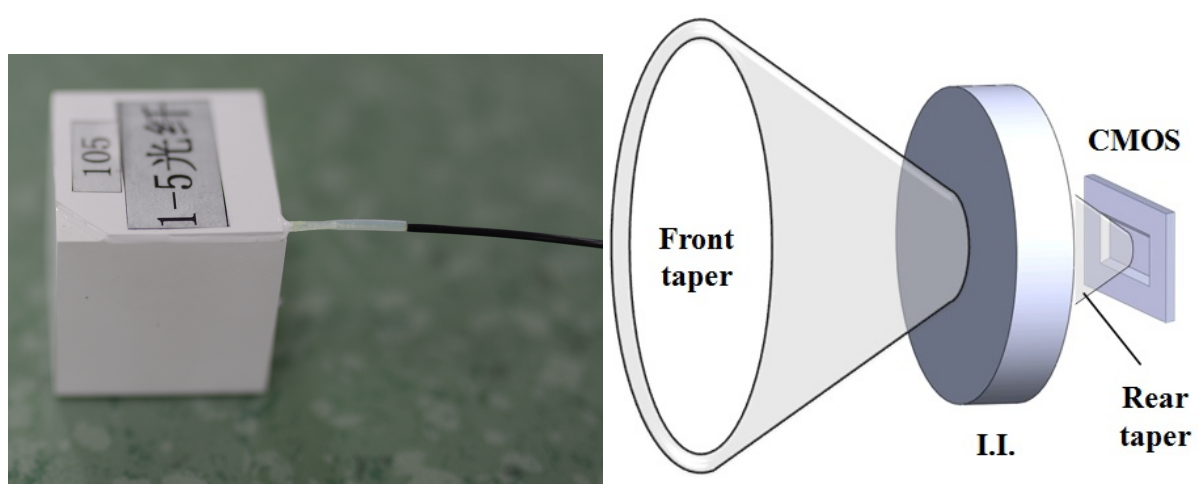

Figure 6: Left: Encapsulation of HERD crystals. All sides are covered by $\mathrm{TiO} 2$ reflection layers. Three WLSFs are routed out. Right: schematic design of IsCMOS system.

\section{References}

[1] Gary Steigman and Michael S. Turner, "Cosmological constraints on the properties of weakly interacting massive particles ", Nuclear Physics B, Volume 253, 1985,375-386.

http://dx.doi.org/10.1016/0550-3213(85)90537-1

[2] Chang, J. and others, "An excess of cosmic ray electrons at energies of 300 - 800GeV", Nature, volume 456, 2008,362-365

[3] Abdo, A. A. and others, "Measurement of the Cosmic Ray $e^{+}+e^{-}$Spectrum from $20 \mathrm{GeV}$ to $1 \mathrm{TeV}$ with the Fermi Large Area Telescope",Phys. Rev. Lett., volume 102, 2009,181101

[4] Aguilar, M. and others, "First Result from the Alpha Magnetic Spectrometer on the International Space Station: Precision Measurement of the Positron Fraction in Primary Cosmic Rays of 0.5ÂíC350 GeV",Phys. Rev. Lett., volume 110, 2013, 141102

[5] "Models of the knee in the energy spectrum of cosmic rays ",Astroparticle Physics, volume 21,2004,241 - 265

[6] "The cosmic-ray experiment KASCADE",Nuclear Instruments and Methods in Physics Research Section A: Accelerators, Spectrometers, Detectors and Associated Equipment, volume $513,2003,490-510$

[7] Amenomori, M. and others, "The All-Particle Spectrum of Primary Cosmic Rays in the Wide Energy Range from $10^{14}$ to $10^{17} \mathrm{eV}$ Observed with the Tibet-III Air-Shower Array",The Astrophysics Journal, volume 678, 2008, 1165-1179

[8] Bartoli, B. and others, "Light-component spectrum of the primary cosmic rays in the multi-TeV region measured by the ARGO-YBJ experiment",Phys. Rev. D, volume 85, 2012, 092005

[9] "Cosmic rays from the knee to the highest energies",Progress in Particle and Nuclear Physics, volume 63, 2009, 293 - 338

[10] "Geant4-a simulation toolkit ",Nuclear Instruments and Methods in Physics Research Section A: Accelerators, Spectrometers, Detectors and Associated Equipment, volume 506, 2003, 250 - 303

[11] Battistoni, G. and others, "The FLUKA code: description and benchmarking",AIP Conference Proceedings, volume 896, 2007, 31-49 
[12] "On the knee in the energy spectrum of cosmic rays ",Astroparticle Physics, volume 19, 2003,193 220

[13] S. Haino,"Precision measurement of the proton flux with AMS",Proc. ICRC,2013

[14] Panov, A. D. and others,"Energy spectra of abundant nuclei of primary cosmic rays from the data of ATIC-2 experiment: Final results",Bulletin of the Russian Academy of Science, Phys. ,volume 73, 2009, 564-567

[15] Shikaze, Y. and others, "Measurements of 0.2 to $20 \mathrm{GeV} / \mathrm{n}$ cosmic-ray proton and helium spectra from 1997 through 2002 with the BESS spectrometer",Astroparticle Physics, volume 28,2007, 154-167

[16] Yoon, Y. S. and others, "Cosmic-ray Proton and Helium Spectra from the First CREAM Flight", The Astrophysics Journal,volume 728, 2011, 122

[17] Ahn, H. S. and others, "Energy Spectra of Cosmic-ray Nuclei at High Energies", The Astrophysical Journal,volume 707, 2009, 593-603

[18] Engelmann, J. J. and others, "Charge composition and energy spectra of cosmic-ray nuclei for elements from Be to NI - Results from HEAO-3-C2",Astronomy and Astrophysics, volume 233, 1990, 96-111

[19] Asakimori, K. and others, "Cosmic-Ray Proton and Helium Spectra: Results from the JACEE Experiment",The Astrophysics Journal,volume 502, 1998, 278-283

[20] Adriani, O. and others, "PAMELA Measurements of Cosmic-Ray Proton and Helium Spectra",Science, volume 332, 2011,69

[21] Derbina, V. A. and others,"Cosmic-Ray Spectra and Composition in the Energy Range of 10-1000 TeV per Particle Obtained by the RUNJOB Experimen",Astrophysical Journal Letters,volume 628, 2005, L41-L44

[22] Ivanenko, I. P. and others,"Energy Spectra of Cosmic Rays above $2 \mathrm{TeV}$ as Measured by the SOKOL Apparatus",International Cosmic Ray Conference, volume 2,1993,17

[23] Ave, M. and others,"Composition of Primary Cosmic-Ray Nuclei at High Energies, The Astrophysics Journal,volume 678, 2008, 262-273

[24] Fang, K. and others, "Discriminating Local Sources of High-Energy Cosmic-Ray Electrons and Positrons by Energy Spectrum and Anisotropy Measurements", arXiv: 1706.03745.

[25] Abdo, A. A. and others, "Fermi Large Area Telescope Observations of the Crab Pulsar and Nebula",Astrophys.J.,volume 708, 2010, 1254-1267

[26] Actis, M. and others,"Design concepts for the Cherenkov Telescope Array CTA: an advanced facility for ground-based high-energy gamma-ray astronomy",Experimental Astronomy, volume 32,2011, 193-316

[27] Zha, M. and LHAASO Collaboration, "Status of the large high altitude air shower observatory project",Nuclear Instruments and Methods in Physics Research A, volume 692, 2012, 77-82

[28] M. Alexander,"CALET Perspectives in High-Energy Gamma-Ray Observations",Proc. ICRC, 2013

[29] Quan, Z., Wang, Z., Xu, M. et al. Radiat Detect Technol Methods (2017) 1: 4. doi:10.1007/s41605-017-0004-4

[30] Y. W. Dong, Z. Quan, J. J. Wang, et al., "Experimental verification of the HERD prototype at CERN SPS", Proc. SPIE 99056D, 2016. doi:10.1117/12.2231804 